\title{
Diversidad de usos de Facebook en la educación superior. Análisis desde un caso de estudio
}

\section{Diversity of uses of Facebook in higher education. Analysis from a case study}

\author{
María Cristina López de la Madrid ${ }^{1,}$ Katiuzka Flores Guerrero ${ }^{2,}$ \\ Adolfo Espinoza de los Monteros Cárdenas ${ }^{3}$ \\ ${ }^{1}$ Departamento de Ciencias Exactas, Tecnología y Metodologías, Universidad de Guadalajara, México \\ (cristilm@ cusur.udg.mx) \\ ${ }^{2}$ Departamento de Ciencias Exactas, Tecnología y Metodologías, Universidad de Guadalajara, México \\ (katiuzka@cusur.udg.mx) \\ ${ }^{3}$ Departamento de Sociedad y Economía, Universidad de Guadalajara, México \\ (adolfoe@ cusur.udg.mx)
}

Recibido el 10 de noviembre de 2015; revisado el 6 de diciembre de 2015; aceptado el 6 de diciembre de 2015 ; publicado el 14 de diciembre de 2015

\section{RESUMEN:}

El uso de las redes sociales ha marcado un momento excepcional dentro de la comunicación entre los humanos. La posibilidad de conectarse con personas de cualquier continente, de una forma rápida y eficaz, es solo uno de los alcances que tienen estas redes, cuyo principal exponente ha sido Facebook, creado en 2004 por un grupo de estudiantes universitarios, y que en 2015 alcanzó un histórico de 1,490 millones de usuarios activos, lo que representa poco más del $20 \%$ de la población mundial. Para conocer los diferentes usos que se le dan a Facebook en la educación superior, se realizó una investigación en el Centro Universitario del Sur (CUSur), de la Universidad de Guadalajara, entre octubre de 2012 y noviembre de 2013. Se trabajaron varias etapas con diferentes actores: docentes, alumnos y Coordinadores de Carrera, buscando con ello tener una representación que integrara los ámbitos de docencia, aprendizaje y administración. Las fases del trabajo fueron las siguientes: se inició con un grupo focal con 9 docentes; posteriormente, se realizaron 10 entrevistas a los Coordinadores de Carrera del CUSur. A partir de algunos de los datos obtenidos, se diseñó una encuesta de reactivos cerrados dirigida a los docentes, y aplicada al $90.5 \%$ de los profesores que en el 2013 señalaron contar con una cuenta de Facebook para trabajo académico (143 docentes). Como una última fase se trabajó un grupo focal con alumnos de diferentes licenciaturas. En la triangulación de los resultados, se observa que los usos en los que coinciden los actores de esta investigación son: comunicación con docentes y alumnos; asesoría y tutorías; circulación de materiales y documentos varios, y actividades de formación y actualización. Se concluye que Facebook puede llegar a tener un importante potencial para enriquecer los procesos educativos, siempre y cuando los docentes, alumnos y administrativos hagan un uso consciente y dirigido de ésta red.

PALABRAS CLAVE: EDUCACIÓN SUPERIOR, IMPACTO DE LA TECNOLOGÍA EN EDUCACIÓN, USOS EDUCATIVOS DE FACEBOOK, CAMBIO EDUCATIVO, REDES SOCIALES.

\section{ABSTRACT:}

The use of the social networks has marked an exceptional moment in communications between humans. The possibility to connect with people from 
every continent, quickly and efficiently, is just one of the achievements that have these networks, whose main exponent has been Facebook, created in 2004 for by a group of university students, and that in 2015 reached a record of 1.490 million active users, representing just over $20 \%$ of the world population. In order to know the different uses that are given to Facebook in higher level education, an investigation was conducted at the Centro Universitario del Sur (CUSur), University of Guadalajara, between October 2012 and November 2013. Several stages with different actors work: teachers, students and educational administrators, thereby seeking to have representation that integrates the areas of teaching, learning and administration. Stages of the study were these: starts with a focus group with nine teachers; subsequently, 10 interviews were conducted with educational administrators. From some of the data, a survey of closed reagents directed to teachers was designed, and applied to $90.5 \%$ of teachers reported that in 2013 have a Facebook account to academic work (143 teachers). As a final phase focus group work with students from different university careers. In the triangulation of the results, it is observed that the uses in agreeing actors of this research are: communication with teachers and students; counseling and mentoring; circulation of materials and various documents, and training and upgrading. We conclude that Facebook can have a significant potential to enrich the educational process, as long as teachers, students and administrative make conscious and aimed use of this network.

\section{KEYWORDS: HIGHER EDUCATION, IMPACT OF TECHNOLOGY ON EDUCATION, EDUCATIONAL USES OF FACEBOOK, EDUCATIONAL CHANGE, SOCIAL NETWORKS.}

\section{ANTECEDENTES}

El uso de las tecnologías de la información y la comunicación (TIC) en educación, es relativamente nuevo; apenas hace 25 años comenzaron las iniciativas de cambio educativo con la inclusión de las TIC en los espacios administrativos y académicos, sobre todo en educación superior. Poco a poco se fueron expandiendo, tanto la diversidad de usos, como los niveles educativos que los implementaban.

En el caso de la red social Facebook, nos encontramos ante un fenómeno cuya expansión ha sido exponencial, y en los últimos cinco años, se han reportado experiencias de su inclusión en la educación con resultados positivos, pero también con avisos de precaución hacia un optimismo amplificado.

Facebook se creó en el 2004 por Mark Zuckerberg y fundado por otros 3 estudiantes de la Universidad de Harvard, cuyo fin era establecer una comunidad virtual al interior de esta universidad. Posteriormente, se fueron integrando estudiantes de otras universidades y en el 2007, Facebook se expandió a otros países; ahora es traducido a 70 idiomas. En el primer trimestre de 2015, superó los 1,370 millones de usuarios activos al mes (Statistic Brain Facebook, 2015). Como señalan Piscitelli y cols. "si Facebook triunfó fue porque se hizo cargo de una necesidad genuina, teniendo como objetivo a una comunidad auténtica" (2010, p. IX).

En México, el número de internautas de 6 años o más, superó el $50 \%$ en el primer trimestre de 2015 , con 53.9 millones de usuarios; la principal actividad en línea, es el acceso a las redes sociales, ya que el 93\% está inscrito en al menos una de ellas. La red social por excelencia es Facebook (Asociación Mexicana de Internet, AMIPCI, 2015). Según datos de la AMIPCI, el 48\% de los usuarios de las redes sociales en Latinoamérica, tienen entre 15 y 24 años de edad.

Las cifras anteriores deben de redundar en investigaciones sobre el impacto de esta red en los espacios sociales. En lo que respecta al espacio educativo, las investigaciones son importantes porque los alumnos utilizan Facebook dentro y fuera de la escuela, desde el nivel básico hasta el posgrado, algunos como medio de comunicación, otros como estrategias colaborativas para enriquecer sus procesos académicos. En este punto, y como menciona Serrat "la formación del estudiante se abre a una multitud de espacios y recursos curriculares sincrónicos y asincrónicos hasta el momento apenas contemplados en las prácticas de la enseñanza universitaria" (2015, p. 44).

Por su parte, el docente se ubica en un espacio estratégico para lograr el cambio que se espera a partir del uso de la tecnología, y atender sus necesidades de infraestructura y de formación y actualización en aspectos relacionados con la tecnología, prácticas pedagógicas y metodologías didácticas, es un imperativo para elevar la calidad educativa a través de espacios virtuales y uso de redes sociales. Rama y Chiecher (2013) identifican el surgimiento de una nueva figura docente, que "despliegan nuevas formas de pensar, sentir y actuar" ante la inclusión de la tecnología en la 
escuela. Como lo señalan Dussel y cols. (2011), en relación a los cambios que se han generado en las políticas educativas "puede señalarse la incorporación al curriculum oficial de materias de tecnología o informática desde hace más de una década, y en los últimos años también al curriculum de la formación docente" (p. 23).

Observamos que los docentes y alumnos, pero también la organización escolar, las prácticas académicas, la normativa, las políticas educativas, los directivos y personal de apoyo, son fuentes de información que pueden aportar datos para la comprensión de las TIC en la educación.

\section{REVISIÓN DE LA LITERATURA}

En las últimas dos décadas, han proliferado estudios relacionados con el uso intensivo de las computadoras e internet, desde lo educativo, social, cultural, económico y político. En la educación han surgido algunas teorías que buscan explicar las transformaciones más importantes en los procesos de enseñanza y aprendizaje, como es el caso del conectivismo (Siemens, 2004), que señala al individuo como punto de partida, y en donde el análisis de redes sociales es un elemento adicional para comprender los modelos de aprendizaje de la era digital. A su vez, se han rescatado y socializado otras teorías que desde décadas anteriores, ponen al alumno en el centro del proceso educativo, como el constructivismo (Piaget y Vygotsky), la teoría del aprendizaje significativo (Ausubel), y el cognoscitivismo, en donde el alumno construye el conocimiento a través de sus estructuras mentales.

Esta recuperación y/o surgimiento de las teorías pedagógicas, conllevan un análisis de las prácticas de enseñanza y aprendizaje más allá de la simple mención de una educación centrada en el alumno. Requiere que los actores educativos se comprometan a modificar sus esquemas para lograr innovaciones que incidan en una mejora de la calidad. Para Serrat (2015, p. 26)

Desde el punto de vista del profesorado, se demanda ahora un nuevo perfil docente que pueda fomentar en el alumnado aprendizajes más significativos, habilidades de pensamiento superior, el aprender a aprender mediante la revisión del ejercicio profesional y la habilidad para el desarrollo del pensamiento reflexivo.
Por su parte, en la investigación realizada por Rama y Chiecher sobre el potencial educativo de Facebook

...fue posible visualizar la emergencia de una nueva figura del alumno universitario, en el marco de los nuevos espacios interactivos virtuales. Esta nueva figura, se construye a partir de la inclusión y participación del estudiante en una significativa práctica educativa, que trasciende los límites del aula y el espacio físico de la universidad, para ser desplegada en un nuevo contexto de interacción, colaboración y construcción de conocimientos entre pares (2013, p.48).

No solo profesores y alumnos deben de modificar sus prácticas educativas; como explican Dussel y Quevedo (2010), "el debate sobre las nuevas tecnologías y su impacto en el sistema educativo debiera partir de la responsabilidad de las políticas públicas, los sistemas educativos y de los adultos respecto a los usos y prácticas que se producen en torno a ellas" (p. 5). Los cambios deben ser tanto personales como organizacionales, desde la escuela pero también desde la perspectiva de los funcionarios de primer nivel, si se quiere lograr una integración de las TIC con resultados positivos para la calidad educativa.

Sin embargo, es importante enfatizar que estos cambios deben de obedecer a paradigmas, estructuras y procesos, más que a cada herramienta tecnológica que vaya saliendo a la luz. Con esto en mente, podemos abordar nuevas aplicaciones desde una lógica diferente, sin tratar de cubrirla con toda una serie de teorías y alcances que durarán hasta la siguiente novedad. Así, para el caso que nos ocupa, el acercamiento a las investigaciones en torno a Facebook, se deriva de la necesidad de una comprensión más amplia dentro de la enseñanza y el aprendizaje.

Como punto de partida, nos interesa saber el nivel de uso de ésta herramienta; según Dussel y Quevedo (2010), en Argentina "durante el año 2009 se duplicó el número de usuarios de las redes sociales y se estimaba que cerca del $81 \%$ de los internautas pertenecían al menos a una red social" (p. 11). Judd (2010) refiere que en un estudio realizado en Estados Unidos en el 2008, se reportó que el $85 \%$ de los estudiantes del nivel superior utilizaba Facebook, comparado con el 14\% de la población general. Este autor señala cómo se ha incrementado el uso de las redes sociales, en comparación con un decrecimiento en la intensidad 
de uso del correo electrónico. En la investigación realizada por Rodríguez y Santamaría en el 2012, identificaron que todas las universidades españolas tienen al menos una cuenta de Facebook como medio de comunicación. Por su parte, Rama y Chiecher (2013) encontraron que "el 92\% de los alumnos encuestados forma parte de una red social y el 83\% forma parte de la red social Facebook" (p. 47).

En otra línea, Gray y cols. (2010) realizaron un estudio sobre el uso de Facebook en estudiantes de Medicina, concluyendo que los docentes pueden contribuir a una mejor inserción profesional de sus estudiantes a través de las redes sociales, al desarrollar en ellos habilidades en el uso de las TIC. Sin embargo, y como explican Espuny y cols.

A pesar del amplio consenso que existe respecto a su utilización [de las redes sociales], de la rapidísima generalización de la mayoría de ellas [...], y de la opinión generalizada de que las potencialidades de su explotación pedagógica es tan amplia como interesante, el aprovechamiento didáctico de esos recursos solo ha tenido experiencias aisladas en la educación universitaria (2011, p. 174).

Percibimos que se ha generalizado la idea de que Facebook es solo una red social para tratar asuntos personales y que poco puede aportar a la educación. En este sentido, y para Llorens y Capdeferro,

El éxito de Facebook como red social, sin embargo, no depende solo de su capacidad para conectar personas, aunque sea esta su orientación inicial. La potencia de la plataforma para compartir recursos, para vincular contenidos presentes en internet a los perfiles de los usuarios y su evolución hacia el lifestreaming y el microblogging la facultan para dar soporte a experiencias de interacción complejas y continuas, y, con ello, para estructurar procesos de aprendizaje colaborativo (2011, p. 31).

En la investigación realizada por Jong et al. (2014), (citado en Esquivel y Rojas, 2014), de los seis motivos conocidos para usar esta red social (mantenimiento de relaciones, pasar el tiempo, mantener comunidades virtuales, entretenerse, mantenerse actual y generar compañerismo), la interacción entre usuarios pertenecientes a la institución educativa fue la cuarta más importante.
A partir de los resultados obtenidos en el estudio ECAR (2012) en relación a los alumnos de licenciatura y las TIC, se identificó la necesidad de balancear las estrategias de innovación con una sólida distribución se servicios institucionales básicos y prácticas pedagógicas y conocer a los estudiantes lo suficientemente bien como para entender cuáles son las innovaciones que más valoran. En este sentido, y como mencionan Pollara y Zhu (2011) "if students are already investing time and e energy in social networking, building relationships and their own communities of shared interest and fostering 21st century skills in the process, there is a unique opportunity to form educational communities of knowledge".

Desde los anteriores elementos, consideramos que toda investigación sobre usos educativos de Facebook aportará elementos para conocer sus potencialidades y diversidad de aplicaciones, así como para identificar posibles distractores en el proceso educativo.

\section{PLANTEAMIENTO DEL PROBLEMA}

Para el caso que nos ocupa, el Centro Universitario del Sur (CUSur), de la Universidad de Guadalajara, se trata de una institución ubicada en el interior del Estado de Jalisco, México, con un área de influencia de 28 Municipios. En el CUSur se imparten 14 licenciaturas, 3 posgrados, 1 Técnico Superior Universitario y 2 Nivel Técnico; cuenta con cerca de 8,000 alumnos y 520 profesores, de los cuales el $24 \%$ son de tiempo completo y el resto de tiempo parcial.

El CUSur tiene un antecedente de 14 años en la implementación de las TIC en los programas académicos y en la administración, a partir de cursos en línea, videoconferencias y materiales audiovisuales para los primeros, y con el uso del Sistema Integral de Información y Administración Universitaria (SIIAU), para lo segundo. En los últimos años se ha intensificado el uso de redes sociales para la realización de diferentes actividades institucionales.

En el 2013, se reportaron 278 docentes con una cuenta de Facebook -de los cuales solo 158 señalaron hacer algún uso educativo-, y 13 de los 14 Coordinadores de Carrera (Excepto Enfermería) reportaron tener grupos abiertos con los alumnos para avisos, reportes y actividades varias; esto fue un indicador para determinar que la mayoría de estos alumnos contaba con una cuenta de Facebook. Cabe aclarar que en 2015 el número de docentes y 
administrativos que utiliza Facebook para actividades laborales supera el $90 \%$.

En diversas páginas de estadísticas y estudios sobre Facebook, se reporta que entre los principales usos es estar en contacto con familiares y amigos, y el encontrar a antiguos conocidos, pero se reportan escasos usos educativos.

A partir de las cifras sobre los usuarios de Facebook en México y en el mundo, y en específico de las características del ámbito de estudio, y de los pocos registros sobre los usos de esta red en los espacios educativos, se ha considerado importante, como objetivo central de este trabajo: Indagar cuáles son los usos de Facebook dentro de la educación en el nivel superior a partir de un caso de estudio.

\section{MÉTODO}

Para la comprensión del objeto de estudio, se trabajó con un enfoque mixto equilibrado, bajo el modelo de Flick (2004, p. 281)

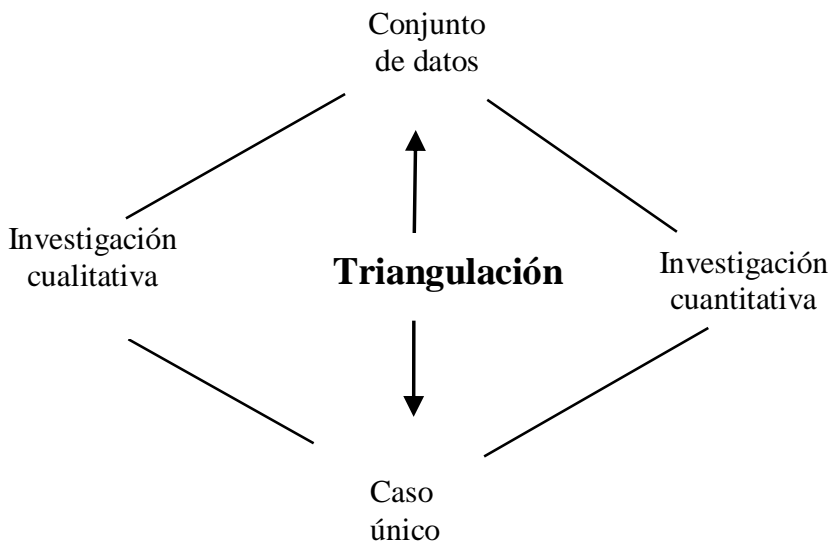

Para este autor, las respuestas de las encuestas y cuestionarios se analizan desde su frecuencia y distribución, y las respuestas a las entrevistas se analizan y comparan para desarrollar una tipología para después unir y comparar ambos grupos de datos.

\subsection{Técnicas de recolección de datos y participantes}

Se trabajaron 3 técnicas: grupo focal, entrevista semiestructurada y encuesta de reactivos cerrados.

a) Se trabajaron dos grupos focales, uno con docentes en donde participaron 9 integrantes de la Academia de Computación, y el segundo con 13 alumnos de las diferentes carreras del Centro Universitario, todos ellos dijeron hacer uso de Facebook tanto para cuestiones personales como académicas. b) Las entrevistas semiestructuradas se realizaron a 10 de los 14 Coordinadores de las Carreras del CUSur. Estos Coordinadores son los encargados de sistematizar y organizar las diferentes acciones relacionadas con los procesos administrativos y educativos de los estudiantes, como: altas y bajas de materias, seguimiento del plan de estudios, seguimiento en Control Escolar, etc. Esta figura es la que está en contacto directo con todos los estudiantes inscritos en un Programa Educativo.

c) La encuesta se aplicó a los 158 docentes que en el 2013 refirieron hacer un uso de Facebook como apoyo a su práctica; se recabaron 143 encuestas respondidas $(90.5 \%)$. Cabe aclarar que en la actualidad (2015) el número de docentes del CUSur que tiene una cuenta de Facebook y hace algún uso educativo, ha aumentado de manera importante.

\subsection{Fases de la investigación}

Las fases de trabajo que se llevaron a cabo a lo largo de 14 meses fueron las siguientes:

a) Se inició con el diseño y trabajo del grupo focal de docentes, en octubre y noviembre de 2012.

b) Se hicieron entrevistas semiestructuradas a los Coordinadores de Carrera en enero y febrero de 2013.

c) A partir de los datos recabados en las 2 primeras fases, entre febrero y julio de 2013 se diseñó, validó y aplicó un instrumento tipo encuesta que se aplicó a través de la plataforma comercial Survey Monkey, la cual se ligó a las cuentas de Facebook de los profesores participantes. Para medir la consistencia interna del instrumento, se calculó el Alfa de Cronbach, obteniendo un valor de .964, considerándose altamente confiable.

d) Como cuarta fase, se trabajó un grupo focal con estudiantes de las diferentes carreras del CUSur en el mes de octubre de 2013.

En este trabajo, se presentan los resultados integrados de estas 4 fases.

\section{RESULTADOS}

Datos generales

Docentes. De los 143 docentes participantes en la encuesta, 71 eran hombres y 72 mujeres; el $45.77 \%$ 
se ubica en el rango de edad de 31 a 40 años, el $13.38 \%$ tienen de 22 a 30 años, y el resto se divide a partes iguales entre los del rango de 41 a 50, y más de 50 años. El 78.87\% tiene más de 6 años en la docencia, y el $60.56 \%$ cuenta con grado de Maestría. El $77.86 \%$ ha estudiado algún programa o curso a distancia, y el $60 \%$ de los encuestados, ha impartido cursos en línea.

Alumnos. De los 13 estudiantes que participaron en el grupo focal, 7 eran hombres y 6 mujeres; el $54 \%$ se ubica en el rango de edad de 18 a 21 años, el $31 \%$ de 22 a 25 años, y el $15 \%$ eran mayores de 25 años. Todos los participantes estaban cursando o habían cursado al menos una de sus asignaturas en línea, y todos ellos hacían un uso frecuente de Facebook, tanto para actividades personales como académicas.

Coordinadores de Carrera. De los 10 Coordinadores que participaron en el estudio, 4 eran hombres y 6 mujeres. Todos ellos tenían al menos una cuenta de Facebook, y 5 de ellos tenían una cuenta exclusiva para sus funciones administrativas.

\section{Usos educativos que convergen en los tres grupos de actores}

Comunicación. Este es uno de los principales atractivos de Facebook; los tres grupos de actores refirieron hacer un uso continuo de la red para mantener una comunicación permanente e inmediata entre: docentes y alumnos, alumnos y docentes, alumnos y alumnos, docentes y docentes y Coordinadores de Carrera y alumnos. En este punto es importante señalar que más del $65 \%$ de los participantes en el estudio señalaron hacer uso de teléfonos inteligentes para conectarse a Facebook, logrando una inmediatez en la comunicación. Aquí observamos que prácticamente no existe la comunicación entre los Coordinadores y docentes y viceversa, ya que para tal efecto se hace uso del correo institucional.

Los alumnos se comunican con sus profesores y compañeros principalmente para: indagar sobre las tareas que dejan los profesores, ponerse de acuerdo en la elaboración y entrega de tareas cuando son en equipo, preguntar a sus compañeros dudas sobre la clase, dar avisos sobre cambios de horario, etc. Como ya se mencionó, la mayoría de profesores del CUSur son de tiempo parcial y acuden a la universidad a impartir sus clases y luego se retiran, por lo que en ocasiones esta es la herramienta más eficaz para contactarlos. Los alumnos refieren que en sus muros sienten la libertad de expresión que no tienen en otros medios de comunicación.
Los docentes se comunican con sus pares y sus alumnos para: trabajo colaborativo, reuniones académicas y avisos varios en el caso de los primeros, y para informar cambios de horario, aula, entregas, etc., en el caso de los segundos. Los docentes aceptan la libertad de expresión de sus alumnos en el portal de Facebook, aunque algunos de ellos refieren tener reglas de comunicación como respeto, uso adecuado de la gramática y no salirse de los temas que se están discutiendo en el momento.

Los Coordinadores de Carrera se comunican con sus pares y con sus estudiantes para información general sobre órdenes de pago, asistencia a eventos académicos y deportivos, programas de becas, ausencia de profesores, cambio de aula y horario, etc. Señalan que Facebook ha sido de gran ayuda para contactar a la mayoría de los estudiantes, ya que están conectados varias horas al día.

Asesoría y tutorías. Los 3 grupos de actores utilizan Facebook para realizar actividades de asesorías y tutorías:

Los alumnos consultan a los docentes sobre dudas de las asignaturas, modificaciones a la planeación y asuntos generales. Algunos de los estudiantes señalaron que trabajar con sus tutores por este medio, les ha resultado más beneficioso, ya que pueden externar sus problemas personales sin tener que estar cara a cara.

En el caso de los docentes, el $88.5 \%$ refiere tener sesiones individuales y grupales de trabajo con los alumnos, lo que incluye aclaración de dudas sobre aspectos específicos de las asignaturas, pero también trabajo tutorial, lo que se ha venido incrementando de manera importante. Cabe señalar que en el CUSur se ha trabajado el Programa Integral de Tutorías desde hace 10 años, a través del cual se han obtenido resultados satisfactorios según lo señalan los alumnos.

Por su parte, los Coordinadores de Carrera han aprovechado esta red para asesorías generales, relacionadas con trámites administrativos, consulta del Plan de Estudios de la Carrera, y situaciones personales de los estudiantes. Estos breves intercambios han sido de gran ayuda para los estudiantes quienes refieren que antes de usar Facebook, tenían que buscar otras fuentes de contacto que muchas veces terminaban en la asistencia al campus.

Compartir y obtener recursos diversos. Esta es una de las acciones que obtiene mayor provecho de esta red social, ya que el tránsito de documentos y materiales es inmediato y se tiene la seguridad de que será revisado por todos. 
En el caso de los docentes, el 78\% de ellos suelen enviar a sus alumnos lecturas complementarias a las estipuladas en el programa, así como ligas de interés actualizadas; también es frecuente el envío de documentos entre docentes (53\%), relacionados a proyectos de investigación, al trabajo de academia y asuntos varios. Estos materiales suelen ser documentos, presentaciones, audiovisuales y videos.

Para los Coordinadores de Carrera, este medio ha facilitado en gran medida el envío de documentos a los estudiantes, desde formatos administrativos, hasta convocatorias de becas e intercambios académicos.

Actividades de formación y actualización. Este apartado tiene dos vertientes: por un lado, se promueven y socializan las acciones de formación y actualización, tanto para los alumnos como para los docentes y administrativos. Esto incluye cursos, talleres, seminarios y conferencias.

Por otro lado, en esta red social se llevan a cabo actividades de formación para los alumnos, como parte de una asignatura; así, en Facebook se han trabajado contenidos educativos específicos, ya sea a través de foros de discusión, o de secuencias específicas que parten de un documento o material audiovisual, junto con instrucciones específicas sobre el análisis a realizar.

En el caso de los docentes, éstos refieren haber seguido videoconferencias y talleres cortos a través de este medio, lo que les permite actualizarse en diferentes campos de su especialidad. El 64\% de los docentes refiere una muy buena evaluación en relación a los beneficios de Facebook en su labor académica.

Los Coordinadores de Carrera señalaron haber participado en al menos una actividad de actualización a través de Facebook, ya sea en relación a su función administrativa, o dentro de su campo docente.

\section{CONCLUSIONES Y DISCUSIÓN}

La concepción generalizada del uso de Facebook, es de un espacio de intercambio social y familiar; algunas de las encuestas sociales que se han realizado en torno a los usos más frecuentes de esta red, incluyen actividades de ocio y recreación, comercio, noticias y deportes, entre otros, pero no se incluyen usos educativos. En la investigación realizada, pudimos constatar que en los espacios universitarios Facebook tiene aplicaciones educativas y administrativas que ayudan a facilitar diferentes tareas, más allá de la inmediatez y facilidad de acceso, características que la han colocado en los primeros lugares de entradas de internet.

Para el caso que nos ocupa, los alumnos refirieron que se les hace más fácil consultar sus muros, que entrar a la plataforma para trabajar y consultar las novedades de sus cursos en línea, sin embargo, también refieren que necesitan de la estructura y organización de éstos últimos para avanzar más eficazmente en los contenidos de sus materias.

Las principales motivaciones de uso educativo que tienen los alumnos para utilizar Facebook, son la versatilidad de la herramienta ya que en un solo espacio pueden hacer uso de diferentes aplicaciones para compartir y comunicarse en los diferentes ámbitos de su vida: social, familiar y escolar, así como la virtualidad que les permite trabajar con sus compañeros y profesores sin importar el tiempo y el espacio. Esto nos lleva a concluir que el uso combinado de Facebook y de las plataformas educativas, puede generar resultados positivos en el proceso de aprendizaje de los alumnos, siempre y cuando se haga la programación adecuada y pertinente.

Los docentes, por su parte, señalaron que logran tener un contacto permanente con sus alumnos a través de ésta red, y esto les ha sido útil para llevar a cabo modificaciones inmediatas en sus cursos sin resentir algún desbalance; algunos de estos cambios incluyen la integración de actividades emergentes, el análisis de un material novedoso o noticias relacionadas con el curso, la continuación de los trabajos en caso de ausencias imprevistas, entre otros.

En el caso de los Coordinadores de Carrera, refieren que Facebook les ha facilitado su trabajo, y los alumnos se han visto beneficiados en la medida en que ya no tienen que estar asistiendo al CUSur para preguntar sobre sus trámites y otras cuestiones administrativas. Este beneficio se hace patente sobre todo en aquellos estudiantes que residen en otros Municipios o en zonas alejadas del campus, lo que se refleja en un importante ahorro de tiempo y dinero.

De manera general, y desde la perspectiva de los actores que participaron en esta investigación, señalamos algunos de los principales cambios que se han percibido con el uso de Facebook en la educación superior.

- Diversificación de las vías de comunicación e inmediatez de la misma.

- Mayor participación de los alumnos que la que se obtiene en los cursos en línea u otras plataformas educativas como apoyo a los cursos presenciales. 
- Los alumnos se vuelven más propositivos, al favorecer acciones de interacción como foros o sesiones informales de trabajo.

- Facilidad de ajuste; permite realizar cambios imprevistos e inmediatos a la planeación académica, sin perjudicar la secuencia de trabajo.

- Es importante destacar que uno de los cambios más notorios relacionados con la integración de Facebook en el ámbito académico, es el de la evaluación, ya que ésta tiene que flexibilizar y considerar las actividades realizadas a través de esta red, lo que puede beneficiar a los alumnos, al demostrar interés y gusto por su uso.

La investigación realizada tuvo algunas limitaciones, derivadas tanto de los objetivos propuestos al inicio de la misma, como del tiempo que se tuvo para realizarla. Estas limitaciones son, a su vez, oportunidades para futuras investigaciones.

- No se hizo una indagación sobre el impacto de Facebook en los procesos de enseñanza y aprendizaje, lo que es un campo de oportunidad para conocer mejor los alcances que puede tener Facebook en el espacio educativo.

- Algunos de los datos presentados no son actuales, ya que como se mencionó, a finales del 2015 el número de usuarios de Facebook en el CUSur ha aumentado considerablemente. Sin embargo, y de acuerdo a una indagación general, no se identifican nuevos usos a los ya presentados en este trabajo.

- Consideramos que hace falta trabajar con una mayor profundidad con algunos docentes, ya que tanto en el grupo focal como en la encuesta, se observó una necesidad de expresar a detalle sus impresiones sobre el uso de Facebook en la escuela, por lo que se planea continuar con ésta investigación.

Como señalan Espuny y cols. (2011, p. 182), “es innegable el impacto que internet ha tenido, está teniendo y parece que va a tener en el desarrollo de redes sociales en diferentes contextos; y, por tanto, educadores e instituciones educativas, des $\neg$ de diferentes ámbitos y etapas formativas, no podemos obviarlas". En el estudio realizado por Pollara y Zhu (2011), los investigadores concluyen que

Additional research is needed to explore the most beneficial design for an edusocial space. Though Facebook has been used for some educational purposes, research could explore the specific kinds of activities that are most beneficial to learners. Using social networking sites, however, is still a controversial issue with most schools blocking the site from students and faculty. Thus, it must also be understood if students can view sites like Facebook as educational spaces and be able to engage in learning activities at appropriate times.

Ante el avance tecnológico, las instituciones educativas tenemos que aprovechar el beneficio de las tecnologías de la información para tratar de elevar la calidad educativa, pero también para mantener el interés y compromiso de los alumnos en su proceso formativo. El gusto por conectarse a Facebook varias horas al día, durante 5 o 7 días a la semana según lo refieren los alumnos que participaron en ésta investigación, es un factor que se debe de aprovechar de la mejor manera posible, con una planeación coherente y una diversificación de herramientas tecnológicas.

\section{REFERENCIAS}

Asociación Mexicana de Internet (AMIPCI) (2015). $11^{\circ}$ Estudio sobre los hábitos de los usuarios de internet en México $2015 . \quad$ Recuperado de https://www.amipci.org.mx/es/estudios.

Dussel, I., Quevedo, L. A., y Santillana, F. (2011). Educación y nuevas tecnologías: los desafios pedagógicos ante el mundo digital. Fundación Santillana. Recuperado de: http://cms.sangari.com/midias/2/111.pdf

EDUCAUSE, Center for Applied Research (2012). ECAR study of undergraduate students and information technology 2012 (Research Report). Lousville, CO: EDUCAUSE. Recuperado de http://net.educause.edu.

Espuny, C., González, J., Lleixà, M., y Gisbert, M. (2011). Actitudes y expectativas del uso educativo de las redes sociales en los alumnos. Revista de Universidad y Sociedad del Conocimiento (RUSC), 8(1), 171-185.

Esquivel, I., y Rojas, C. (2014). Uso de Facebook en ámbitos educativos universitarios: Consideraciones y recomendaciones. Apertura, 6(2).

Flick, U. (2004). Introducción a la investigación cualitativa. Madrid: Morata.

Gray, K., Annabelle, L., y Kennedy, G., (2010). Medical students' use of Facebook to support learning: Insights from four case studies. Medical Teacher, 32, 971 - 976.

Judd, T. (2010). Facebook versus email. British Journal of Educational Technology, 41(5) E101-E103, doi: 10.1111/j.1467-8535.2009.01041.x

Junco, R. (2011). The relationship between frequency of Facebook use, participation in Facebook activities, and student engagement. Computer and Education, 58, 162171. doi:10.1016/j.compedu.2011.08.004.

Llorens, F., y Capdeferro, N. (2011). Posibilidades de la plataforma Facebook para el aprendizaje colaborativo en línea. Revista de Universidad y Sociedad del Conocimiento (RUSC), 8(2), 31-45.

Piscitelli, A., Adaime, I. y Binder, I. (Comp.) (2010). El Proyecto Facebook y la posuniversidad. Sistemas operativos sociales y entornos abiertos de aprendizaje. Barcelona: Ariel.

Pollara, P. y Zhu, J. (2011). Social Networking and Education: Using Facebook as an Edusocial Space. Proceedings of 
Society for Information Technology y Teacher Education International Conference. Recuperado de https://www.academia.edu/394232/Social_Networking_ and_Education_Using_Facebook_as_an_Edusocial_Spa ce.

Rama, M., y Chiecher, A. (2013). El potencial educativo de Facebook en la universidad. Contextos de Educación, 12(13), 46-55. Recuperado de http://www.hum.unrc.edu.ar/publicaciones/contextos/art iculos/vol13/rama.html.

Rodríguez, A., y Santamaría, P. (2012). Análisis del uso de las redes sociales en internet. Revista de comunicación y tecnologías emergentes 20(2), 228-246. doi: 10.7195/ri14.v10i2.198

Serrat, N. (2015). Metodologías participativas y Facebook en el ámbito universitario. InnoEduca, l(1), 25-32. Recuperado de http://revistas.uma.es/index.php/innoeduca/article/view/ 104.

Siemens, G. (2004). Connectivism: A Learning Theory for the Digital Age. Recuperado de http://www.elearnspace.org/Articles/connectivism.htm.

Statistic Brain (2015). Facebook statistics. Recuperado de http://www.statisticbrain.com/facebook-statistics/

i Este trabajo se derivó del Proyecto de Investigación "Estudio sobre el uso de Facebook en el nivel superior", financiado a partir de las Convocatorias 2012 y 2013 del Centro Universitario del Sur, de la Universidad de Guadalajara, México. 